\title{
Rate of surgery in patients under treatment with a Chêneau light brace using the SRS inclusion criteria
}

\author{
M Werkmann ${ }^{1 *}$, HR Weiss ${ }^{2}$ \\ From 8th International Conference on Conservative Management of Spinal Deformities and SOSORT 2011 \\ Annual Meeting \\ Barcelona, Spain. 19-21 May 2011
}

\section{Background}

Studies investigating the outcome of conservative scoliosis treatment differ widely with respect to the inclusion criteria used. Prospective cohort studies are available using the SRS inclusion criteria for studies on bracing $[1,2]$. This seems to provide a great advantage to compare different strategies of bracing against each other. As we have gathered all data of the patients treated with a Chêneau light ${ }^{\mathrm{TM}}$ between June 2005 and November 2007 it was possible to identify the sample of patients fulfilling the SRS inclusion criteria from the whole sample.

\section{Materials and methods}

34 patients (of 152) fulfilled the SRS inclusion criteria with an average age of 12.06 years ( $10-13$ years), average Cobb angle of 31 degrees $\left(25-40^{\circ}\right)$, an average Risser stage of 0,35 , average in-brace Cobb angele of $13^{\circ}(=59 \%$ of in-brace correction). There were 17 thoracic, 10 double major, 6 lumbar and 2 thoracolumbar curve patterns. After change of workplace of the second author the patients could not be followed up as planned. Therefore a telephone interview was performed by the first author.

\section{Results}

28 patients (average age 16.5 years) have been reached, 9 of them were still under treatment. No patient has been operated (Rate of surgery $0 \%$ ) and only one was not satisfied with cosmetic outcome of treatment.

\section{Discussion}

Rate of surgery was far less than reported in recent studies using the same inclusion criteria even when all drop outs where rated as failures $[1,2]$.

\section{Conclusions}

Rate of surgery can be reduced with the help of Chêneau braces of the latest standard and satisfactory inbrace correction.

\section{Author details \\ ${ }^{1}$ Scoliocare Orthomed, Gensingen, Germany. ${ }^{2}$ Orthopedic Rehabilitation Services, Gensingen, Germany.}

Published: 27 January 2012

\section{References}

1. Coillard C, Vachon V, Circo AB, Beauséjour M, Rivard CH: Effectiveness of the SpineCor brace based on the new standardized criteria proposed by the scoliosis research society for adolescent idiopathic scoliosis. J Pediatr Orthop 2007, 27(4):375-9.

2. Zaborowska-Sapeta K, Kowalski IM, Kotwicki T, Protasiewicz-Fałdowska H, Kiebzak W: Effectiveness of Chêneau brace treatment for idiopathic scoliosis: prospective study in 79 patients followed to skeletal maturity. Scoliosis 2011, 6(1):2.

doi:10.1186/1748-7161-7-S1-045

Cite this article as: Werkmann and Weiss: Rate of surgery in patients under treatment with a Chêneau light brace using the SRS inclusion criteria. Scoliosis 2012 7(Suppl 1):045.

${ }^{1}$ Scoliocare Orthomed, Gensingen, Germany

Full list of author information is available at the end of the article 